\title{
Acute Intracranial Hemorrhage in CT: Benefits of Sinogram-Affirmed Iterative Reconstruction Techniques
}

\author{
B. Bodelle, E. Klein, N.N.N. Naguib, R.W. Bauer, J.M. Kerl, F. Al-Butmeh, J.L. Wichmann, H. Ackermann, T. Lehnert, T.J. Vogl, and B. Schulz
}

\begin{abstract}
BACKGROUND AND PURPOSE: Acute intracranial hemorrhage represents a severe and time critical pathology that requires precise and quick diagnosis, mainly by performing a CT scan. The purpose of this study was to compare image quality and intracranial hemorrhage conspicuity in brain CT with sinogram-affirmed iterative reconstruction and filtered back-projection reconstruction techniques at standard (340 mAs) and low-dose tube current levels (260 mAs).
\end{abstract}

MATERIALS AND METHODS: A total of 94 consecutive patients with intracranial hemorrhage received CT scans either with standard or low-dose protocol by random assignment. Group 1 ( $n=54$; mean age, $64 \pm 20$ years) received CT at $340 \mathrm{mAs}$, and group 2 ( $n=40$; mean age, $57 \pm 23$ years) received CT at $260 \mathrm{mAs}$. Images of both groups were reconstructed with filtered back-projection reconstruction and 5 iterative strengths (S1-S5) and ranked blind by 2 radiologists for image quality and intracranial hemorrhage on a 5-point scale. Image noise, SNR, dose-length product (mGycm), and mean effective dose (mSv) were calculated.

RESULTS: In both groups, image quality and intracranial hemorrhage conspicuity were rated subjectively with an excellent/good image quality. A higher strength of sinogram-affirmed iterative reconstruction showed an increase in image quality with a difference to filtered back-projection reconstruction $(P<.05)$. Subjective rating showed the best score of image quality and intracranial hemorrhage conspicuity achieved through S3/S4-5. Objective analysis of image quality showed in an increase of SNR with a higher strength of sinogramaffirmed iterative reconstruction. Patients in group 2 (mean: $744 \mathrm{mGycm} / 1.71 \mathrm{mSv}$ ) were exposed to a significantly lower dose than those in group 1 (mean: $1045 \mathrm{mGycm} / 2.40 \mathrm{mSv}, P<.01$ ).

CONCLUSIONS: S3 provides better image quality and visualization of intracranial hemorrhage in brain CT at $260 \mathrm{mAs}$. Dose reduction by almost one-third is possible without significant loss in diagnostic quality.

ABBREVIATIONS: DLP = dose-length product; $\mathrm{FBP}=$ filtered back-projection reconstruction; $\mathrm{HU}=$ Hounsfield units; $\mathrm{ICH}=$ intracranial hemorrhage; $\mathrm{SAFIRE}=$ sinogram-affirmed iterative reconstruction

A t present, CT of the brain is the imaging technique of choice for evaluation of an intracranial hemorrhage (ICH). CT imaging adds valuable information regarding the extent and severity of an ICH. Every effort should be made to accurately detect ICH because of the higher mortality rate without treatment.

CT examinations account for only a minority of radiologic procedures but represent a significant portion of the radiation

Received March 18, 2013; accepted after revision July 8.

From the Departments of Diagnostic and Interventional Radiology (B.B., E.K N.N.N.N., R.W.B., J.M.K., F.A.-B., J.L.W., T.L., T.J.V., B.S.) and Biostatistics and Mathematical Modeling (H.A.), Goethe University, Frankfurt, Germany.

Paper previously presented in part at: European Congress of Radiology, March 7-11, 2013; Vienna, Austria.

Please address correspondence to Boris Bodelle, MD, Department of Diagnostic and Interventional Radiology, Goethe University, Theodor-Stern-Kai 7, 60590 Frankfurt, Germany; e-mail: bbodelle@googlemail.com

Evidence-Based Medicine Level 1.

http://dx.doi.org/10.3174/ajnr.A3801 dose received from all medical procedures. ${ }^{1-6}$ Because of the potential radiation risk through ionizing radiation and because $\mathrm{CT}$ is frequently in use for patients with head trauma, every effort should be made to keep the dose as low as reasonably achievable.

Many approaches to reduce patient dose have been investigated including routine use of automated exposure control software, and reduction of tube current and tube potential. Reducing the tube current is eventually limited by increased noise leading to a decrease in image quality. Recently, iterative reconstruction techniques for CT have been introduced to decrease image noise as an alternative to the standard filtered back-projection (FBP) method. ${ }^{7-11}$ Earlier versions of iterative reconstruction algorithms required a high amount of computational calculating time and could not be used in this form for emergency radiologic procedures. ${ }^{12}$ The second generation of iterative reconstruction processes, sinogram-affirmed iterative reconstruction (SAFIRE), is now commercially available. SAFIRE estimates the noise content 

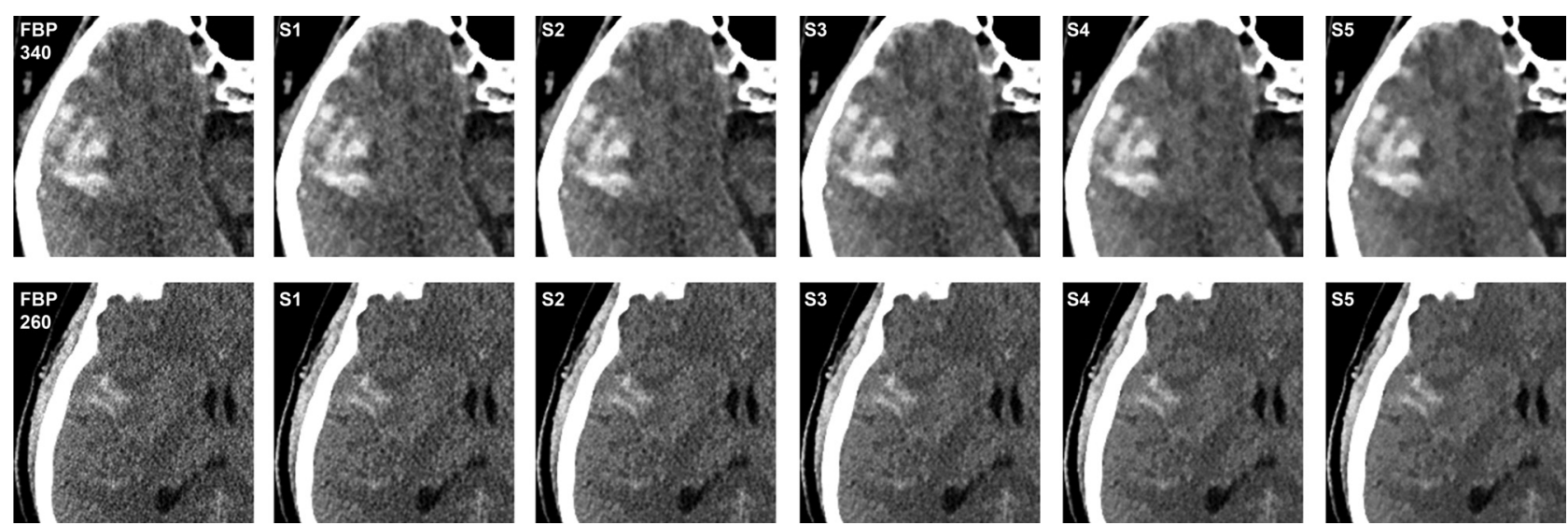

FIG 1. CT images of a 67-year-old man with intracerebral bleeding acquired with tube current-time product of $340 \mathrm{mAs}$ (upper row) and a 48-year-old man with subarachnoid hemorrhage acquired with tube current-time product of $260 \mathrm{mAs}$ (lower row), each with FBP and 5-strength of the SAFIRE technique.

in raw data caused by fluctuations in neighboring voxels and subtracts the noise stepwise in several validation loops. The result of the first correction loop is compared with the "master data," and an updated image is generated for the next iteration, leading to further noise reduction. Offered by various vendors, this technique should be able to reduce the necessary radiation dose by $35 \%-76 \%$ while maintaining equivalent image quality. ${ }^{13-15}$ For this purpose, we conducted this study to compare the SAFIRE algorithm and FBP regarding image quality and detectability of $\mathrm{ICH}$ and reduction of radiation dose in brain CT scanning.

\section{MATERIALS AND METHODS \\ Patient Selection}

This study was approved by our institutional review board. The data analyzed in this study were acquired in a timeframe of 8 months. In this timeframe, all patients referred for CT of the brain were examined either with the standard CT protocol (group 1) or with the new protocol with reduced mAs (group 2) by random assignment. All consecutive patients with ICH were included: 54 patients from group 1 and 40 patients from group 2.

\section{Examination Techniques}

All patients underwent the examination on multidetector row CT scanners (Somatom Definition Flash/AS; Siemens, Erlangen, Germany). CT protocol settings followed the manufacturer's recommendations. The parameters were kept constant, except the tube current-time products; $340 \mathrm{mAs}$ was the standard scan parameter and $260 \mathrm{mAs}$ was the new parameter for this study, as recommended by the manufacturer. The CT was acquired in axial image orientation, $48-\mathrm{mm}$ detector coverage $(4 \times 20 \times 0.6 \mathrm{~mm})$, a small field of view, and $120 \mathrm{kVp}$. Images from the top to the base of the anterior cranial fossa were evaluated.

\section{Image Reconstruction}

Images of both tube current levels ( $340 \mathrm{mAs}$ and $260 \mathrm{mAs}$ ) were reconstructed with a medium smooth kernel (H30s, J30s) into $4-\mathrm{mm}$ sections by use of FBP and 5 different blending strengths of SAFIRE (S1-S5), which led to a total of 6 image datasets as shown in Fig 1. All image sets were sent for processing to a PACS workstation (Centricity 4.1; GE Healthcare, Dornstadt, Germany).

\section{Dose Estimates}

For the estimation of radiation doses, we recorded the doselength product (DLP in $\mathrm{mGycm}$ ) and the effective tube currenttime product (effective mAs) from the patient protocol, which is automatically generated after the end of an examination and stored in the PACS of our department. The effective dose was calculated as the product of DLP and the normalized value of effective dose per DLP for the head $(0.0023 \mathrm{mSv}$ mGy-1 cm-1; European guideline on quality criteria for CT, European Commission, EUR 16262).

\section{Subjective Image Quality}

All CT image datasets were displayed in random order on a diagnostic monitor for the assessment of subjective image quality and with all images displayed on constant window settings (window width, 80 Hounsfield units [HU]; window level, $40 \mathrm{HU}$ ). These datasets were reviewed by 2 radiologists with experience in neuroradiology in a blinded manner. The overall image quality of brain structures and the image quality and identifiable properties of cerebral hemorrhage were ranked by use of a 5 -point scale ( $1=$ worst image quality, 2 = fair image quality, $3=$ moderate image quality, $4=$ good image quality, $5=$ best image quality). The subjective image quality ratings from FBP and SAFIRE S1-S5 at each level of tube current-time product were compared with standard FBP at $340 \mathrm{mAs}$ and FBP at $260 \mathrm{mAs}$.

\section{Objective Analysis of Image Quality}

As measures of image quality, 4 ROI measurements were performed on a PACS workstation by use of a circle tool with a diameter of 3-6 mm for the ROIs. The measurements were performed by a radiologist with 1 year of experience in CT of the brain. Image noise $(I N)$ was determined as the standard deviation of air in the level of the frontal lobe. Mean attenuation values $(A)$ and standard deviation were measured in the GM and WM in the superior frontal gyrus and in the center of the ICH: cerebral, epidural, subdural, and subarachnoid hemorrhage. The measurements for 6 image datasets of each patient were recorded and displayed in HU. On the basis of these measurements, SNR was determined according to the following equation:

$$
S N R=A / I N
$$


Table 1: Subjective image quality rating $(1=$ worst, $5=$ best $)$ for brain structures and intracranial hemorrhage for filtered back-projection and 5 strengths (S1-S5) of sinogram-affirmed iterative reconstruction technique

\begin{tabular}{|c|c|c|c|c|c|c|c|c|}
\hline \multirow{3}{*}{$\begin{array}{c}\text { Image Reconstruction } \\
\text { Technique }\end{array}$} & \multicolumn{4}{|c|}{ Rating Brain Structures } & \multicolumn{4}{|c|}{ Rating Intracranial Hemorrhage } \\
\hline & \multicolumn{2}{|c|}{$340 \mathrm{mAs}$} & \multicolumn{2}{|c|}{$260 \mathrm{mAs}$} & \multicolumn{2}{|c|}{$340 \mathrm{mAs}$} & \multicolumn{2}{|c|}{$260 \mathrm{mAs}$} \\
\hline & & $\kappa$ & & $\boldsymbol{\kappa}$ & & $\boldsymbol{\kappa}$ & & $\kappa$ \\
\hline FBP & $2.8 \pm 0.4$ & 0.71 & $2.0 \pm 0.8$ & 0.59 & $2.5 \pm 0.7$ & 0.9 & $2.1 \pm 0.8$ & 0.67 \\
\hline S1 & $3.5 \pm 0.6^{\mathrm{a}}$ & 0.71 & $2.9 \pm 0.6^{a}$ & 0.48 & $3.15 \pm 0.8^{c}$ & 0.85 & $2.8 \pm 0.8^{\mathrm{a}, \mathrm{c}}$ & 0.9 \\
\hline S2 & $4.4 \pm 0.6$ & 0.77 & $3.7 \pm 0.7^{\mathrm{a}}$ & 0.51 & $3.8 \pm 0.7^{c}$ & 0.82 & $3.4 \pm 0.7^{c}$ & 0.75 \\
\hline S3 & $4.5 \pm 0.5^{c}$ & 0.47 & $4.7 \pm 0.5^{c}$ & 0.62 & $4.39 \pm 0.7^{c}$ & 0.80 & $4.0 \pm 0.7^{c}$ & 0.72 \\
\hline S4 & $3.2 \pm 0.7^{\mathrm{a}, \mathrm{c}}$ & 0.86 & $3.4 \pm 1.0^{\mathrm{a}, \mathrm{c}}$ & 0.55 & $4.65 \pm 0.6^{c}$ & 0.81 & $4.65 \pm 0.5^{c}$ & 0.61 \\
\hline S5 & $2.0 \pm 0.7^{b, c}$ & 0.64 & $2.5 \pm 0.9^{b, c}$ & 0.37 & $4.44 \pm 0.7^{c}$ & 0.81 & $4.68 \pm 0.6^{c}$ & 0.55 \\
\hline
\end{tabular}

Note:- ${ }^{\text {a }} P>.05$ when compared with standard FBP at $340 \mathrm{mAs} ;{ }^{b} P>.05$ when compared with FBP at 260 mAs; ${ }^{c} P>0.1$ when compared with the same image reconstruction technique at different tube current-time product.

Interobserver agreement (slight $[\kappa<0.3]$, moderate $[\kappa=0.3-0.7]$, good agreement $[\kappa>0.7]$ ).

Table 2: Objective image quality measurements with SNR and image noise for filtered back-projection and 5 strength (S1-S5) of sinogram-affirmed iterative reconstruction technique

\begin{tabular}{|c|c|c|c|c|c|c|c|c|}
\hline \multirow{2}{*}{$\begin{array}{c}\text { Image Reconstruction } \\
\text { Technique }\end{array}$} & \multicolumn{2}{|c|}{ SNR White Matter } & \multicolumn{2}{|c|}{ SNR Gray Matter } & \multicolumn{2}{|c|}{ SNR Intracranial Hemorrhage } & \multicolumn{2}{|c|}{ Image Noise } \\
\hline & $340 \mathrm{mAs}$ & $260 \mathrm{mAs}$ & $340 \mathrm{mAs}$ & $260 \mathrm{mAs}$ & $340 \mathrm{mAs}$ & $260 \mathrm{mAs}$ & $340 \mathrm{mAs}$ & $260 \mathrm{mAs}$ \\
\hline FBP & $15.9 \pm 4.8^{\mathrm{b}, \mathrm{c}}$ & $15.0 \pm 2.9^{\mathrm{a}, \mathrm{c}}$ & $25.1 \pm 6.5^{b}$ & $21.0 \pm 3.8^{\mathrm{a}}$ & $38.2 \pm 11.6^{b, c}$ & $34.4 \pm 7.4^{\mathrm{a}, \mathrm{c}}$ & 1.76 & 1.97 \\
\hline S1 & $19.4 \pm 5.6^{\mathrm{a}, \mathrm{c}}$ & $17.0 \pm 3.1^{a, b, c}$ & $29.9 \pm 6.9$ & $23.7 \pm 3.8^{\mathrm{a}, \mathrm{b}}$ & $45.6 \pm 12.8$ & $39.0 \pm 7.6^{\mathrm{a}, \mathrm{b}}$ & 1.42 & 1.72 \\
\hline S2 & $21.9 \pm 5.7^{c}$ & $18.9 \pm 3.6^{\mathrm{a}, \mathrm{c}}$ & $34.0 \pm 6.6$ & $26.6 \pm 4.8^{\mathrm{a}}$ & $52.1 \pm 13.9^{c}$ & $43.6 \pm 9.6^{a, c}$ & 1.21 & 1.55 \\
\hline S3 & $23.9 \pm 6.9$ & $20.8 \pm 4.1$ & $37.7 \pm 9.1$ & $29.1 \pm 5.3$ & $57.1 \pm 16.5$ & $47.9 \pm 10.1$ & 1.16 & 1.41 \\
\hline S4 & $26.0 \pm 6.8$ & $22.7 \pm 4.6$ & $41.1 \pm 9.6$ & $31.7 \pm 6.1$ & $63.2 \pm 19.3$ & $52.3 \pm 11.2$ & 1.03 & 1.30 \\
\hline S5 & $29.9 \pm 7.3$ & $25.4 \pm 5.8$ & $46.5 \pm 9.4$ & $35.5 \pm 7.5$ & $71.2 \pm 20.2$ & $58.4 \pm 13.4$ & 0.89 & 1.17 \\
\hline
\end{tabular}

Note:- ${ }^{a} P>.05$ for SNR when compared with standard FBP at $340 \mathrm{mAs} ;{ }^{b} P>.05$ for SNR when compared with FBP at $260 \mathrm{mAs} ;{ }^{c} P>.05$ when compared with the same image reconstruction technique at different tube current-time product $(\mathrm{mAs}=$ tube current $\times$ seconds).

The objective image quality ratings from FBP and SAFIRE S1-S5 at each level of tube current-time product were compared with standard FBP at $340 \mathrm{mAs}$ and FBP at $260 \mathrm{mAs}$.

\section{Statistical Analysis}

Computer-based statistical analyses were performed with dedicated software (BiAS 9.17; Epsilon, Frankfurt, Germany). Patient age, image noise, SNR, subjective image quality, and DLP were expressed as mean values and standard deviations. Age and DLP were tested by use of the Wilcoxon-Mann-Whitney $U$ test. For SNR and subjective image quality rating, the Fisher exact test with a Bonferroni-corrected $P$ value was used. A $P$ value of $<5 \%$ was considered to be statistically significant. Interobserver agreement of subjective image quality rating was assessed with the Cohen weighted $\kappa$ analysis. Definitions of levels of agreement on the basis of $\kappa$ values were as follows: $\kappa<0.3$ indicated slight agreement; $\kappa=0.3-0.7$, moderate agreement; and $\kappa>0.7$ meant good agreement.

\section{RESULTS}

\section{Patient Characteristics}

In group 1 with the standard protocol, 54 patients with a mean age of $64 \pm 20$ years (age range, 17-90 years) were included. In group 2 , with a reduced tube current-time product of $260 \mathrm{mAs}, 40$ patients with a mean age of $57 \pm 23$ years (age range, 16-95 years) were included. No significant differences regarding age were found concerning patient characteristics.

\section{Subjective Image Quality Measurements}

Subjective image quality was rated for FBP and SAFIRE S1-S5 at each level of tube current-time product. Data are summarized in Table 1. Subjective overall image quality of brain structures was rated with excellent interobserver agreement for both readers for group 1 at $340 \mathrm{mAs}(\kappa=0.91)$ and group 2 with $260 \mathrm{mAs}(\kappa=$ 0.80 ). In a likewise fashion, the image quality with visibility of ICH in group 1 and group 2 was rated subjectively with a good interobserver agreement $(\kappa=0.92$ and $\kappa=0.9$, respectively). The score for image quality of brain structures increased with a higher strength of SAFIRE at each level of tube current-time product up to $\mathrm{S} 3$ and for image quality of ICH up to S4-S5. The best score for the image quality of brain structures was achieved with SAFIRE S3 with significant difference to the FBP reconstruction in each group $(P<.05)$. The best score for the visualization of ICH was achieved with SAFIRE S4 and S5 with significant difference to the FBP reconstruction in each group $(P<.05)$. The difference between standard FBP at $340 \mathrm{mAs}$ and SAFIRE 1 blending at 260 $\mathrm{mAs}$ had no predominant statistical significance for image quality of the brain structures and ICH $(P>.05)$. When compared with 1 reconstruction technique on its own (FBP or SAFIRE S1-S5) between both tube current-time products, there was no statistically significant difference $(P>.1)$ for S3-S5 depicting brain structures and for S1-S5 depicting ICH conspicuity.

\section{Objective Image Quality Measurements}

Statistical results of the objective image quality measurements are summarized in Table 2. Image noise was higher $(P>.05)$ in group $2(260 \mathrm{mAs})$ than in group $1(340 \mathrm{mAs})$ for all reconstruction techniques. Image noise decreased with higher strength of SAFIRE; therefore, SNR increased with a higher strength of SAFIRE at each level of tube current-time product ( $\mathrm{mAs}$ ). The difference between FBP and SAFIRE 1 was statistically significant $(P<.05)$ for SNR GM and ICH at $340 \mathrm{mAs}$, whereas no significant difference was reached for examination at $260 \mathrm{mAs}$. No statistically significant difference was shown for SNR WM at SAFIRE 1 for both groups. SNR of SAFIRE 3 up to 5 at $260 \mathrm{mAs}$ and $340 \mathrm{mAs}$ 
was higher than FBP at each level of tube current-time product $(P<.05)$.The highest SNR was reached with SAFIRE 5 in each group. When compared with 1 reconstruction technique on its own (FBP or SAFIRE S1-S5) between both tube current-time products, there was no statistically significant difference $(P>.05)$ for FBP, S1 and S2 depicting WM, and FBP and S2 depicting ICH.

\section{Radiation Dose}

Patients examined with a tube current of $260 \mathrm{mAs}$ were exposed to significantly less radiation dose than the group examined with 340 mAs (260 mAs: mean DLP, $744 \pm 80$ mGycm; 340 mAs: mean DLP, $1045 \pm 108 \mathrm{mGycm} ; P<.01)$. Even the calculated mean effective dose was lower at $1.71 \mathrm{mSv}$ (260 mAs) compared with $2.40 \mathrm{mSv}$ (340 mAs).

\section{DISCUSSION}

Previous studies have applied the standard deviation to assess the objective image quality. ${ }^{8,16}$ To assess the objective image quality in the brain and to compare the level of the signal with the level of background noise, we applied the SNR of selected ROIs to WM and GM and the region with ICH as a measurement of objective image quality. Ren et $\mathrm{al}^{17}$ showed a possible reduction of the tube current-time product down to $200 \mathrm{mAs}$ on CT of the brain with the adaptive statistical iterative reconstruction technique without focus on ICH. Because of ethical reasons, we followed the manufacturer's recommendations. With reduction of the tube currenttime product from the standard $340 \mathrm{mAs}$ down to $260 \mathrm{mAs}$, the mean effective dose decreased from $2.40-1.71 \mathrm{mSv}$, which results in a relevant reduction of $29 \%$, higher than the $20.4 \%$ calculated by Korn et al, ${ }^{18}$ and similar to Ren et al ${ }^{17}$ and Kilic et al ${ }^{19}$ with $30 \%$ and $31 \%$ dose reductions, respectively.

Compared with the FBP reconstruction technique, all iterative reconstruction techniques increased SNR heterogeneously by $13 \%-88 \%$, depending on the algorithm strength and tube current-time product. These results are similar to those of Schulz et $\mathrm{al}^{12}$ for CT of the paranasal sinus and Leipsic et $\mathrm{al}^{9}$ for coronary CT angiography. There was no significant difference for SNR between FBP and SAFIRE S1 for group 2 at $260 \mathrm{mAs}$. Therefore, we concluded that SAFIRE 1 could not provide a better noise reduction than FBP in lower-dose examination at $260 \mathrm{mAs}$. We came to the conclusion that SAFIRE 3-5 is able to reduce noise and increase objective image quality in standard brain CT even with a lower tube current-time product.

SAFIRE 3 was rated best for overall image quality of the brain at both $\mathrm{mAs}$ levels, with the best $\kappa$ value of 0.62 for examination at $260 \mathrm{mAs}$. SAFIRE 4-5 was rated best for the visualization of ICH, and we conclude that the higher noise reduction leads to a better demarcation of lesions with rich contrast. The use of the SAFIRE 1 reconstruction technique with a reduced tube current-time product of $260 \mathrm{mAs}$ could achieve the same image quality as a standard examination at $340 \mathrm{mAs}$. SNR at $340 \mathrm{mAs}$ is still higher than in low-dose examination at $260 \mathrm{mAs}$, but it does not affect radiologic diagnosis significantly. For standard use, we recommend a protocol at $260 \mathrm{mAs}$ with an iterative algorithm. The benefit of higher SNR at $340 \mathrm{mAs}$ can be assumed for postoperative or therapeutic cerebral status. An appropriate examination protocol should be reserved for patients with a relevant medical history.

Although the SNR increases with a higher strength of SAFIRE, the subjective image quality with SAFIRE S4 and S5 is worse than with SAFIRE S3. We deduce that a high SAFIRE strength does not necessarily imply a good image quality. This phenomenon is similar to what has been reported in previous studies. Increased image blurring has been discussed in several publications that investigated iterative reconstruction techniques. ${ }^{9,12,14,20,21}$ Silva et $\mathrm{al}^{20}$ suggested that the diminished noise manifests as an oversmoothing of the images. Singh et $\mathrm{al}^{8}$ thought that higher blending proportions of iterative reconstruction to FBP could substantially change the texture and characteristics of the images. As discussed before, SAFIRE offers a better reconstruction technique for the detection of ICH than FBP, and it can be assumed that a better reconstruction technique with SAFIRE reconstruction leads to a lower false-negative value of the detection of $\mathrm{ICH}$, better treatment, and a possible reduction of radiation risk. Although the $\kappa$ value varied for the subjective image qualities, the variation tendency of the 2 radiologists was consistent, probably because of different diagnostic experience and different understanding about the scales. These differences cannot be avoided, but in a randomized and blinded manner, they can be minimized.

For the future, iterative algorithms should be used for the detection of ICH. This should be accompanied by a lowering of the false-negative rate for the detection of ICH. SAFIRE 3 is the choice for evaluation of brain structures and SAFIRE 5, the choice for evaluation of ICH at both mAs levels. Inexperienced readers are recommended to use SAFIRE 3 for evaluation of the brain structures and SAFIRE 5 for evaluation and detection of ICH in combination. To optimize the processing time, experienced readers should rely on the SAFIRE 3 algorithm at $260 \mathrm{mAs}$ for evaluation of the brain structures and detection of $\mathrm{ICH}$ with 1 reconstruction algorithm. The benefit of SAFIRE 5 compared with SAFIRE 3 concerning the conspicuity of ICH would not affect radiologic diagnosis significantly for experienced readers.

There were limitations to our study. First, we could not perform an intrapatient comparison, but we had 2 groups with no significant difference regarding age, and all patients were referred for assessment of ICH. Second, the measurement by ROI did not provide information for the whole brain. However, with essential ROIs we received valid results as evidence for the subjective image quality in the whole brain. For future studies, SAFIRE can be used in combination with other dose-reduction techniques, for instance, automatic tube-current modulation, as proposed by Smith et $\mathrm{al}^{22}$ as an effective dose-reduction method or automatic tube-voltage modulation.

\section{CONCLUSIONS}

SAFIRE improves image quality and visualization of ICH on head CT with a normal-dose and a low-dose protocol. For standard use, we recommend a protocol at $260 \mathrm{mAs}$ with a SAFIRE algorithm with the benefit of a reduction in radiation dose by approximately $29 \%$. For evaluation in patients with postoperative or therapeutic cerebral status, a benefit at $340 \mathrm{mAs}$ can be assumed. SAFIRE 3 showed an increased image quality for evaluation of brain structures and SAFIRE 5 for ICH conspicuity compared 
with the FBP reconstruction technique at both $\mathrm{mAs}$ levels. The use of SAFIRE 3 at $260 \mathrm{mAs}$ is recommended for evaluation of brain structures and detection of $\mathrm{ICH}$, optimizing processing time for experienced readers. For unexperienced readers, SAFIRE 5 at $260 \mathrm{mAs}$ should be used for evaluation of ICH in combination with SAFIRE 3 for evaluation of brain structures. SAFIRE should be used to diminish the false-negative rate. Therefore, a better detection with an iterative algorithm can result in better treatment.

Disclosures: Ralf Bauer-UNRELATED: Payment for Lectures (including service on speaker bureaus): Seimens AG. J. Matthias Kerl—UNRELATED: Payment for Lectures (including service on speaker bureaus): Siemens AG. J. Matthias Kerl and Ralf Bauer are consultants for Siemens Medical Care.

\section{REFERENCES}

1. Linton OW, Mettler FA Jr. National conference on dose reduction in CT, with an emphasis on pediatric patients. AJR Am J Roentgenol 2003;181:321-29

2. Yates SJ, Pike LC, Goldstone KE. Effect of multislice scanners on patient dose from routine CT examinations in East Anglia. $\mathrm{Br} \mathrm{Ja}$ diol 2004;77:472-78

3. Brix G, Nagel HD, Stamm G, et al. Radiation exposure in multi-slice versus single-slice spiral CT: results of a nationwide survey. Eur Radiol 2003;13:1979-91

4. Brenner DJ, Hall EJ. Computed tomography-an increasing source of radiation exposure. $N$ Engl J Med 2007;357:2277-84

5. Wiest PW, Locken JA, Heintz PH, et al. CT scanning: a major source of radiation exposure. Semin Ultrasound CT MR 2002;23:402-10

6. Schenzle JC, Sommer WH, Neumaier K, et al. Dual energy CT of the chest: how about the dose? Invest Radiol 2010;45:347-53

7. Vandenberghe S, D'Asseler Y, Van de Walle R, et al. Iterative reconstruction algorithms in nuclear medicine. Comput Med Imaging Graph 2001;25:105-11

8. Singh S, Kalra MK, Gilman MD, et al. Adaptive statistical iterative reconstruction technique for radiation dose reduction in chest $\mathrm{CT}$ : a pilot study. Radiology 2011;259:565-73

9. Leipsic J, Labounty TM, Heilbron B, et al. Adaptive statistical iterative reconstruction: assessment of image noise and image quality in coronary CT angiography. AJR Am J Roentgenol 2010;195:649-54

10. Hara AK, Paden RG, Silva AC, et al. Iterative reconstruction tech- nique for reducing body radiation dose at CT: feasibility study. $A J R$ Am J Roentgenol 2009;193:764-71

11. Flicek KT, Hara AK, Silva AC, et al. Reducing the radiation dose for CT colonography using adaptive statistical iterative reconstruction: A pilot study. AJR Am J Roentgenol 2010;195:126-31

12. Schulz B, Beeres M, Bodelle B, et al. Performance of iterative image reconstruction in $\mathrm{CT}$ of the paranasal sinuses: a phantom study. AJNR Am J Neuroradiol 2013;34:1072-76

13. Funama $Y$, Taguchi K, Utsunomiya D, et al. Combination of a lowtube-voltage technique with hybrid iterative reconstruction (iDose) algorithm at coronary computed tomographic angiography. J Comput Assist Tomogr 2011;35:480-85

14. Bulla S, Blanke P, Hassepass F, et al. Reducing the radiation dose for low-dose CT of the paranasal sinuses using iterative reconstruction: feasibility and image quality. Eur J Radiol 2012;81:2246-50

15. Pontana F, Duhamel A, Pagniez J, et al. Chest computed tomography using iterative reconstruction vs filtered back projection (Part 2): image quality of low-dose CT examinations in 80 patients. Eur Radiol 2011;21:636-43

16. Birnbaum BA, Hindman N, Lee J, et al. Multi-detector row CT attenuation measurements: assessment of intra- and interscanner variability with an anthropomorphic body CT phantom. Radiology 2007;242:109-19

17. Ren Q, Dewan SK, Li M, et al. Comparison of adaptive statistical iterative and filtered back projection reconstruction techniques in brain CT. Eur J Radiol 2012;81:2597-601

18. Korn A, Fenchel M, Bender B, et al. Iterative reconstruction in head $\mathrm{CT}$ : image quality of routine and low-dose protocols in comparison with standard filtered back-projection. AJNR Am J Neuroradiol 2012;33:218-24

19. Kilic K, Erbas G, Guryildirim M, et al. Lowering the dose in head CT using adaptive statistical iterative reconstruction. AJNR Am J Neuroradiol 2011;32:1578-82

20. Silva AC, Lawder HJ, Hara A, et al. Innovations in CT dose reduction strategy: application of the adaptive statistical iterative reconstruction algorithm. AJR Am J Roentgenol 2010;194:191-99

21. Mitsumori LM, Shuman WP, Busey JM, et al. Adaptive statistical iterative reconstruction versus filtered back projection in the same patient: 64 channel liver CT image quality and patient radiation dose. Eur Radiol 2012;22:138-43

22. Smith AB, Dillon WP, Gould R, et al. Radiation dose-reduction strategies for neuroradiology CT protocols. AJNR Am J Neuroradiol 2007;28:1628-32 